\title{
Implantation of a collagen matrix for an AMIC repair during dry arthroscopy
}

\author{
Boguslaw Sadlik • Martin Wiewiorski
}

Received: 21 September 2013/Accepted: 2 May 2014/Published online: 13 May 2014

(C) Springer-Verlag Berlin Heidelberg 2014

\begin{abstract}
Several arthroscopic cartilage repair techniques in the knee joint require the insertion of a cut-to-shape matrix. Depending on the location and accessibility of the cartilage lesion, this procedure can be challenging. To overcome the limitations of currently used techniques, a novel matrix insertion technique was developed. This technique utilizes an inserter rod and a dedicated guide which can be used in a dry arthroscopy setup.

Level of evidence Expert opinion, Level V.
\end{abstract}

Keywords Cartilage repair - Knee - Patella - AMIC . Arthroscopy · Matrix

\section{Introduction}

Several advanced cartilage repair techniques utilize natural or synthetical matrices to restore hyaline (-like) cartilage with improved biomechanical properties [5]. Examples of such techniques are third generation ACI [7] or matrix

Electronic supplementary material The online version of this article (doi:10.1007/s00167-014-3062-x) contains supplementary material, which is available to authorized users.

\section{B. Sadlik}

Orthopaedic Department, St Luke's Clinic, ul. Bystrzanska 94B, 43-309 Bielsko-Biala, Poland

e-mail: bs@lukasza.pl

M. Wiewiorski ( $\square)$

Center for Advanced Orthopedic Studies, Beth Israel, Deaconess Medical Center, Harvard Medical School, Boston, MA, USA

e-mail: wiewiorskim@gmail.com

M. Wiewiorski

Orthopaedic Department, University of Basel Hospital, Basel, Switzerland augmented intrinsic bone marrow repairs such as autologous matrix-induced chondrogenesis (AMIC) [1], or hyaluronan-based scaffold implantation (Hyalofast) [9]. The trans-arthroscopic insertion of matrices to the knee joint surface can be challenging. Some limiting factors are the presence of arthroscopic fluid and poor accessibility due to confined space (patello-femoral joint). Additionally, soft collagen matrices tend to coil, fold up, or displace, which complicates insertion of such implants in a conventional arthroscopy setup. Therefore, most authors choose to perform matrix insertion in a mini-open procedure. An allarthroscopic solution is favourable. To simplify arthroscopic collagen-matrix insertion, a novel system utilizing an insertion rod and a corresponding guide tool has been developed.

\section{Technical note}

The insertion of the matrix demonstrated in this section follows the principles of the AMIC procedure for a lateral patellar cartilage lesion [1]. In the AMIC procedure, the cartilage defect is debrided, followed by bone marrow stimulation, and subsequent insertion of a bilayer matrix composed of porcine type I/III collagen (Chondro-Gide, Geistlich Pharma AG, Wolhusen, Switzerland) [1]. The surgery begins with a conventional arthroscopy (Video 1). The choice of portals depends on the lesion location. Standard anterolateral, lateral, and suprapatellar lateral portals are installed. Once the patellar lesion is located, an intra-articular retraction system to increase the work space in the joint and improve access to the patellar lesion is used. For this procedure, a retraction plate is inserted through the arthroscopic portals and placed next to the patella. Thick sutures attached to the end of each plate run 

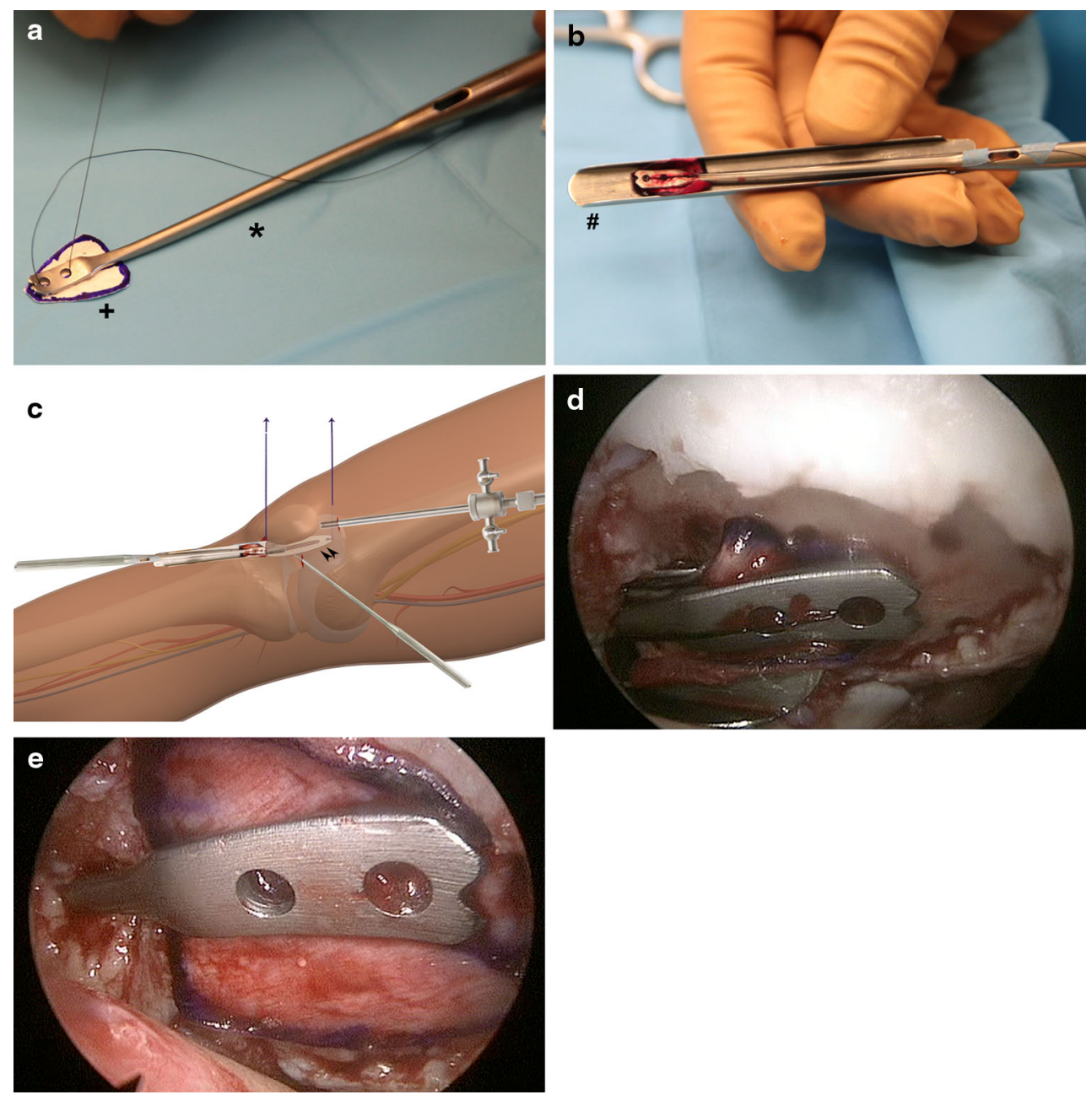

Fig. 1 Matrix preparation and insertion. The matrix $(+)$ is attached to the inserter $\operatorname{rod}(*)$ with a 2-0 non-resorbable thread that runs through two holes at the tip of the rod (a). In this case, the matrix was immersed in bone marrow concentrate and the inserter rod was placed on the insertion guide $(\#, \mathbf{b})$. The rod with the guide were inserted through the anterolateral portal and placed near the defect on the

through the portals to the outside of the joint and are attached to a holder rod. Tension applied to the sutures lifts the plate subsequently distracting the joint cavity and tilting the patella. Additionally, the retraction plate prevents the joint cavity from collapsing after evacuation of the saline solution. The defective cartilage is debrided. In order to detect lesion size, the minimum and maximum diagonal of the defect is measured. The results are then transferred to the matrix, which is cut to size. Microdrilling can be performed antegrade through the joint cavity or retrograde by transpatellar drilling. Before the matrix can be inserted, intra-articular fluid needs to be evacuated to create a dry environment. The prepared matrix is immersed in saline solution or bone marrow concentrate. For insertion of the matrix, two instruments are needed: an inserter rod with a flat tip and two holes (Fig. 1a) to accommodate the matrix patella $(\mathbf{c})$. In this case, a retraction plate $(\rightarrow)$ was used to lift and tilt the patella to gain adequate access. After evacuation of the saline solution from the joint, the matrix was positioned over the defect by pushing the inserter rod over the guide (d). Once the matrix was in place (d), the thread was cut and the rod and guide were removed

and a guide (Fig. 1b) to channel the rod with the matrix through an arthroscopic portal into the joint cavity. The matrix is sutured to the tip of the rod with a non-resorbable 2-0 suture (Fig. 1a). The matrix is then immersed in saline solution or bone marrow concentrate. The guide is inserted into an arthroscopic portal that allows straight access to the lesion (Fig. 1c). The inserter rod with the matrix slides through the guide into the joint cavity and is placed over the debrided and microfractured lesion (Fig. 1d). Once positioned correctly, the sutures holding the matrix to the rod can be cut outside of the joint (Fig. 1e). The matrix is fixated with fibrin glue along the edges of the defect. Typically, the fibrin glue needs 5 min to dry. During this time, the matrix has to be pressed against the bottom of the defect. This can be done with an arthroscopy hook in small lesions, or with a Foley catheter balloon in cases of lesions 
larger than $150 \mathrm{~mm}^{2}$ [13]. With the matrix in place, the knee joint is moved several times through its range of motion. The matrix should remain in place. It is important that the matrix remains stable at the cartilage interface. If not, additional fibrin glue should be added.

\section{Discussion}

Successful trans-arthroscopic implantation of matrices in cartilage repair procedures has been previously described $[3,6,10]$. However, an all-arthroscopic approach in a conventional fluid filled joint can be challenging with soft collagen matrices, like the one used for the AMIC -procedure. In order for adequate access and easy matrix handling, an additional mini-open arthrotomy is commonly performed to aid the AMIC technique [4]. A minimally invasive approach avoiding disruption of the joint capsule and the patellar stabilizing ligaments would be favourable. This would also reduce the risk for arthrofibrosis.

In the presented novel surgical technique, the collagen matrix is sutured to an inserter rod and guided into the joint cavity. This technique allows straight forward insertion in an all-arthroscopy setup, avoiding common issues like folding and coiling of the soft matrix. Displacement of the matrix into the joint cavity is avoided, preventing a painstaking and time-consuming recovery procedure. The technical pearls and pitfalls are given in Table 1.

Another important prerequisite for successful transarthroscopic matrix insertion is adequate access to the lesion. Specifically, in confined spaces like the patello-femoral joint, an additional retracting device is recommended to create enough work space for matrix handling. Several retraction techniques have been described [8, 12]. The authors favour a minimally invasive retraction system consisting of an intra-articular retraction plate, which can be used to lift the patella, preventing the joint from collapsing. Once adequate work space in the knee joint is established, the arthroscopic fluid is evacuated creating a dry joint cavity

Table 1 Pearls and pitfalls of matrix-aided cartilage repair in a dry arthroscopy setup

\begin{tabular}{lc}
\hline Pearls & Pitfalls \\
\hline $\begin{array}{l}\text { Appropriate planning of portal } \\
\text { location is essential: wrong } \\
\text { portal site complicates surgery }\end{array}$ & $\begin{array}{c}\text { Exact implant size is hard to } \\
\text { judge arthroscopically } \\
\text { Implant folding/rolling is time } \\
\text { and extends surgery time }\end{array}$ \\
$\begin{array}{cc}\text { One can always switch to a mini- } \\
\text { open approach, if arthroscopy }\end{array}$ & $\begin{array}{c}\text { Fogging of the arthroscope in a } \\
\text { dry arthroscopy setup is } \\
\text { fails }\end{array}$ \\
$\begin{array}{ll}\text { In cases of a ruptured retraction } \\
\text { suture, the plate can be easily } \\
\text { recovered from the joint }\end{array}$ \\
\hline
\end{tabular}

[11]. In our opinion, a fluid or gas-free joint cavity is recommended for use of soft collagen matrices, as described above for the AMIC procedure. Furthermore, the dry arthroscopy setup facilitates fibrin glue application onto the lesion and matrix, enabling the glue to harden faster. Overall, fibrin glue fixation is time saving, technically easy to perform and does not affect matrix integrity [2].

\section{Conclusion}

Arthroscopic matrix insertion in a dry arthroscopy setup utilizing the presented inserter rod and guide is a feasible and safe procedure for AMIC treatment of knee cartilage lesions.

Acknowledgments The authors thank Christie-Joy Cunningham for her help in the correcting of this article, Rafal Szopa for providing the schemes for the figures, and the Medicalmovies.eu team for help in editing of the video material.

\section{References}

1. Behrens P (2005) Matrixgekoppelte Mikrofrakturierung. Arthrosk 18:193-197

2. Bekkers JE, Tsuchida AI, Malda J, Creemers LB, Castelein RJ, Saris DB, Dhert WJ (2010) Quality of scaffold fixation in a human cadaver knee model. Osteoarthritis and cartilage/OARS. Osteoarthr Res Soc 18(2):266-272

3. Brittberg M, Concaro S (2011) Autologous chondrocyte implantation : transarthroscopic implantation of hyalograft (Hyaff 11) with autologous chondrocytes. Cartilage surgery. Elsevier, Philadelphia, pp 147-160

4. de Girolamo L, Quaglia A, Bait C, Cervellin M, Prospero E, Volpi P (2012) Modified autologous matrix-induced chondrogenesis (AMIC) for the treatment of a large osteochondral defect in a varus knee: a case report. Knee Surg Sports Traumatol Arthrosc 20(11):2287-2290

5. Filardo G, Kon E, Roffi A, Di Martino A, Marcacci M (2013) Scaffold-based repair for cartilage healing: a systematic review and technical note. Arthroscopy 29(1):174-186

6. Fontana A (2012) A novel technique for treating cartilage defects in the hip: a fully arthroscopic approach to using autologous matrix-induced chondrogenesis. Arthrosc Tech 1(1):e63-e68

7. Knutsen G, Drogset JO, Engebretsen L, Grontvedt T, Isaksen V, Ludvigsen TC, Roberts S, Solheim E, Strand T, Johansen O (2007) A randomized trial comparing autologous chondrocyte implantation with microfracture. Findings at five years. J Bone Joint Surg Am 89(10):2105-2112

8. Maeno S, Hashimoto D, Otani T, Masumoto K, Matsumoto H, Enomoto H, Niki Y, Yuzawa I, Fukui Y, Ishikawa M, Fujita N, Okubo M (2008) Hanger-lifting procedure in knee arthroscopy. Arthroscopy 24(12):1426-1429

9. Marcacci M, Berruto M, Brocchetta D, Delcogliano A, Ghinelli D, Gobbi A, Kon E, Pederzini L, Rosa D, Sacchetti GL, Stefani G, Zanasi S (2005) Articular cartilage engineering with Hyalograft C: 3-year clinical results. Clin Orthop Relat Res 435:96-105

10. Piontek T, Ciemniewska-Gorzela K, Szulc A, Naczk J, Slomczykowski M (2012) All-arthroscopic AMIC procedure for 
repair of cartilage defects of the knee. Knee Surg Sports Traumatol Arthrosc 20(5):922-925

11. Sadlik B, Wiewiorski M (2014) Dry arthroscopy with a retraction system for matrix-aided cartilage repair of patellar lesions. Arthrosc Tech 3(1):e141-e144

12. Siebold R, Sartory N, Yang Y, Feil S, Paessler HH (2011) Prone position for minimal invasive or all-arthroscopic autologous chondrocyte implantation at the patella. Knee Surg Sports Traumatol Arthrosc 19(12):2036-2039

13. Wood D, Janes GC (2011) Autologous chondrocyte implantation : matrix-induced autologous chondrocyte implantation (MACI). Cartilage surgery. Elsevier, Philadelphia, pp 161-173 